\title{
Charge pumping and the colored thermal voltage noise in spin valves
}

\author{
Jiang Xiao, ${ }^{1}$ Gerrit E.W. Bauer, ${ }^{1}$ Sadamichi Maekawa, ${ }^{2,3}$ and Arne Brataas ${ }^{4}$ \\ ${ }^{1}$ Kavli Institute of NanoScience, Delft University of Technology, 2628 CJ Delft, The Netherlands \\ ${ }^{2}$ Institute for Materials Research, Tohoku University, Sendai, Japan \\ ${ }^{3}$ CREST, Japan Science and Technology Agency, Tokyo 100-0075, Japan \\ ${ }^{4}$ Department of Physics, Norwegian University of Science and Technology, NO-7491 Trondheim, Norway
}

\begin{abstract}
Spin pumping by a moving magnetization gives rise to an electric voltage over a spin valve. Thermal fluctuations of the magnetization manifest themselves as increased thermal voltage noise with absorption lines at the ferromagnetic resonance frequency and/or zero frequency. The effect depends on the magnetization configuration and can be of the same order of magnitude as the Johnson-Nyquist thermal noise. Measuring colored voltage noise is an alternative to ferromagnetic resonance experiments for nano-scale ferromagnetic circuits.
\end{abstract}

\section{INTRODUCTION}

A spin valve consists of a thin non-magnetic metallic layer (NM) sandwiched by two ferromagnetic layers (FM) with variable magnetization direction. One of the FM layers is usually thick and its magnetization is fixed, while the other is thin and its magnetization direction is free to move. Spin valves have a wide range of interesting static and dynamic properties, $, 1,2,3,4,5,6,7,8,9,10,11,12,13$ many of which are related to the current-induced spin-

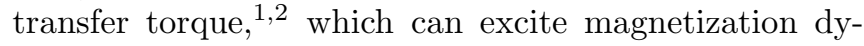
namics (and reversal). Inversely, magnetization dynamics generates a current flow or a voltage output. Berger first discussed the induced voltage in an FM|NM|FM structure by magnetization dynamics $\underline{14}$ He posited that a voltage of order $\hbar \omega / e$ can be generated when the magnetization of one ferromagnet precesses at frequency $\omega$. Similar dynamically induced voltages have been studied theoretically 15 and observed ${ }^{16}$ in simple $\mathrm{FM} \mid \mathrm{NM}$ junctions and in magnetic tunnel junctions (MTJs) 17 In spin valves and MTJs, voltage induced by the magnetization dynamics can be understood as a two-step process: i) the moving magnetization of the free layer generates a spin current; ii) the static magnetization of the fixed layer filters the "pumped" spin current and converts it into a charge current or, in an open circuit, a voltage output. The electrical voltage induced by moving domain walls can be explained analogously: $\frac{18,19,20,21,22}{1 n}$ the first part of the present paper, we derive a simple formula for the charge pumping voltage in a spin valve by circuit theory in which magnetization dynamics is taken into account. We find that the magnitude of the voltage is governed by the spin-transfer torque in the same structure. We therefore propose to measure the angular dependence of the spin-transfer torque (or torkance, i.e. the torque divided by the voltage bias) by the angular dependence of the charge pumping voltage.

The charge pumping voltage consists of a DC and an AC component, even when induced by a steady magnetization dynamics such as ferromagnetic resonance (FMR). The concept can be extended to the case of thermally activated, i.e. fluctuating, magnetization dynamics, which is an extra source of thermal voltage noise that only appears in magnetic structures. Johnson ${ }^{23}$ and Nyquist ${ }^{24}$ showed that in non-magnetic conductors the voltage noise is associated with thermal agitation of charge carriers (driven by fluctuating electromagnetic modes). The power spectrum of this noise is white and proportional to the temperature $T$ and resistance $R: S_{J N}(\omega)=4 k_{\mathrm{B}} T R$ up to frequencies of $k_{\mathrm{B}} T / \hbar \sim 10^{4}$ $\mathrm{GHz}$ at room temperature ${ }^{23,24}$ In magnetic structures such as spin valves thermal fluctuations of the magnetization direction have to be considered $\stackrel{25}{\underline{w}}$ Some consequences of thermal fluctuation in spin valves, such as noise-facilitated magnetization switching $26,27,28$ and resistance fluctuations,$\stackrel{29,30}{=}$ have been studied before. In the so called thermal ferromagnetic resonance, frequencies are studied by means of resistance fluctuations without applied magnetic fields $\stackrel{31}{\underline{ }}$ Foros et al ${ }^{30}$ have shown that the time-averaged auto-correlator of the resistance fluctuations is significantly affected by dynamical exchange coupling between the magnetic layers.

In the second part of this paper, we show that a magnetization fluctuation-related voltage noise can be of the same order of magnitude as the conventional thermal noise in non-magnetic conductors. This noise is not "white" but displays spectral features related to the ferromagnetic resonance (FMR). The noise spectrum therefore contains information comparable to that obtained by FMR. For nano-scale ferromagnetic circuits the noise measurements might be easier to perform than conventional FMR experiments. Compared to the resistance noise measurement, the pumping voltage noise measurement is non-intrusive because it does not require application of current, which may disturb the system.

The paper is organized as follows: Section II presents a general theoretical frame work that combines the magnetoelectric circuit theory and the Landau-Lifshitz-Gilbert (LLG) equation. In Section III, we derive a formula for the charge pumping voltage in spin valves. In Section IV, by using the magnetic susceptibility function, we calculate the voltage noise spectrum due to magnetization fluctuations for two different magnetic configurations. Section V contains some general remarks on the calculation. In Appendix A we calculate the angular-dependence of the magnetic susceptibility for a 
spin valve, and Appendix B presents an alternative calculation of the magnetization-related thermal noise by computing the frequency dependent impedance of a spin valve.

\section{CIRCUIT THEORY WITH DYNAMICS}

Fig. 1(a) shows a spin valve structure under consideration. The magnetization in the left FM with direction $\mathbf{m}_{0}$ is assumed to be static and $\mathbf{m}$, the one of the right FM, to be free, which can be realized by making the right layer much thinner than the left one. For electron transport, we assume for simplicity that the spin valve is symmetric. Such an assumption may be invoked when both FM layers are of the same material and thicker than the spin flip diffusion length. In that regime, the resistances of the bulk ferromagnet over the spin-flip diffusion length are in series with the interface resistances, whereas the remoter parts of the ferromagnets are magnetically inert series resistances. The regime in which the layers become thinner than the spin flip diffusion length has been treated by Kovalev et. al ${ }^{32}$ In this and the next Section, we focus on the spin-active region in the spin valve, which includes the NM spacer and small part (of the order of the spin-flip diffusion length) of the FM layers as indicated by the dashed box in Fig. 1(a).

When $\mathbf{m}$ depends on time, a spin current is pumped into the metallic spacer layer through the $\mathrm{F} \mid \mathrm{N}$ interface. The magnitude and polarization of the spin pumping current reads: ${ }^{33}$

$$
\mathbf{I}_{s}^{s p}=\frac{\hbar}{4 \pi}\left(g_{r} \mathbf{m} \times \frac{d \mathbf{m}}{d t}+g_{i} \frac{d \mathbf{m}}{d t}\right),
$$

where $g_{r}$ and $g_{i}$ are the real and imaginary part of the dimensionless transverse spin-mixing conductance $\stackrel{33}{3}$ The

(a) driven magnetization motion

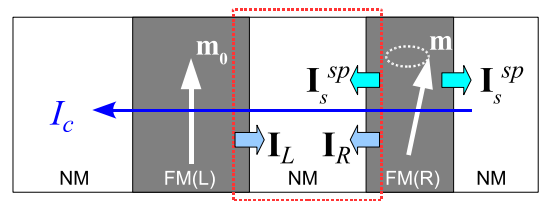

(b) thermal magnetization fluctuations

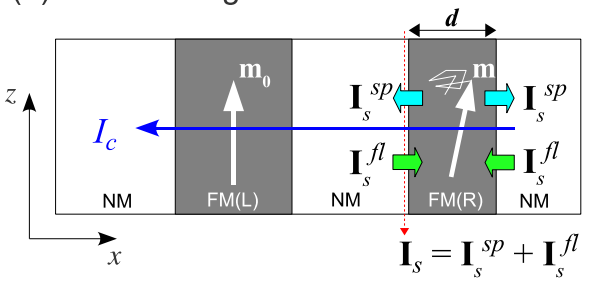

FIG. 1: (Color online) Spin and charge currents in spin valves. (a) For the steady state case studied in Section [I] and III (b) For the thermal magnetization fluctuations studied in Section IV. first term in $\mathbf{I}_{s}^{s p}$ corresponds to a loss of angular momentum of the free layer magnetization to the adjacent NM layers, thus providing an extra damping torque ${ }^{33}$ When the adjacent normal metal is an ideal spin sink, the spin pumping current loss can be represented by a Gilbert damping coefficient (see below), and each interface contributes to the damping constant by $\alpha^{\prime}=\left(\gamma \hbar / 4 \pi M_{t o t}\right) g_{r}$, introducing the gyromagnetic ratio $\gamma$ and the total magnetization of the right FM film $M_{t o t}$. The imaginary part $g_{i}$ effectively modifies the gyromagnetic ratio for the magnetization under consideration.

In order to use magnetoelectronic circuit theory, the structure has first to be decomposed into nodes (for bulk) and contacts (for interfaces). For each node we may define a charge chemical potential and a (vector) spin chemical potential: let $\mu_{L, R, N}$ and $\boldsymbol{\mu}_{L, R, N}$ be the charge and spin chemical potentials in left, right FM leads, and the NM spacer. In the ferromagnet, we may assume that the spin accumulations are aligned with the magnetization, i.e. $\boldsymbol{\mu}_{L}=\mu_{L}^{s} \mathbf{m}_{0}$ and $\boldsymbol{\mu}_{R}=\mu_{R}^{s} \mathbf{m}$. The charge current $I_{c}$ and the spin current $\mathbf{I}_{L}$ through the left interface connecting the left FM and the spacer layer are given by: $\stackrel{34,35}{=}$

$$
\begin{aligned}
I_{c} & =\frac{e g}{2 h}\left[2\left(\mu_{L}-\mu_{N}\right)+p\left(\mu_{L}^{s}-\boldsymbol{\mu}_{N} \cdot \mathbf{m}_{0}\right)\right], \\
\mathbf{I}_{L} & =-\frac{e g}{2 h}\left[2 p\left(\mu_{L}-\mu_{N}\right)+\left(\mu_{L}^{s}-\boldsymbol{\mu}_{N} \cdot \mathbf{m}_{0}\right)\right] \mathbf{m}_{0} \\
& +\frac{e}{h} g_{r} \mathbf{m}_{0} \times \boldsymbol{\mu}_{N} \times \mathbf{m}_{0}+\frac{e}{h} g_{i} \boldsymbol{\mu}_{N} \times \mathbf{m}_{0},
\end{aligned}
$$

where $g=g_{\uparrow}+g_{\downarrow}$ is the total conductance and $p=$ $\left(g_{\uparrow}-g_{\downarrow}\right) / g$ is the polarization of the $\mathrm{F} \mid \mathrm{N}$ interface and the (longitudinal) active regions of the FMs $\underline{32}$ Similarly for the right FM lead:

$$
\begin{aligned}
I_{c} & =-\frac{e g}{2 h}\left[2\left(\mu_{R}-\mu_{N}\right)+p\left(\mu_{R}^{s}-\boldsymbol{\mu}_{N} \cdot \mathbf{m}\right)\right], \\
\mathbf{I}_{R} & =-\frac{e g}{2 h}\left[2 p\left(\mu_{R}-\mu_{N}\right)+\left(\mu_{R}^{s}-\boldsymbol{\mu}_{N} \cdot \mathbf{m}\right)\right] \mathbf{m} \\
& +\frac{e}{h} g_{r} \mathbf{m} \times \boldsymbol{\mu}_{N} \times \mathbf{m}+\frac{e}{h} g_{i} \boldsymbol{\mu}_{N} \times \mathbf{m}+\frac{2 e}{\hbar} \mathbf{I}_{s}^{s p},
\end{aligned}
$$

where the spin current $\mathbf{I}_{R}$ at the right interface is modified due to the additional spin pumping current $\mathbf{I}_{s}^{s p}$ emitted by the moving magnetization $\mathbf{m}$. Additionally, the spin current conservation in the presence of the spin flips in the NM spacer requires:

$$
\mathbf{I}_{L}+\mathbf{I}_{R}=\frac{e}{h} \frac{h}{D \tau_{s f}} \boldsymbol{\mu}_{N} \equiv \frac{e}{h} g_{s f} \boldsymbol{\mu}_{N},
$$

where $D$ the energy density of states at the Fermi energy and $\tau_{s f}$ the spin flip relaxation time in the NM.

The spin current $\mathbf{I}_{R}$ entering the free layer exerts a spin-transfer torque on $\mathbf{m}$, which is equal to its transverse component absorbed at the interface: 11,12

$$
\left.\mathbf{N}_{\mathrm{st}}=\frac{\hbar}{2 e}\left[\mathbf{I}_{R}-\left(\mathbf{I}_{R} \cdot \mathbf{m}\right) \mathbf{m}\right)\right]
$$


The LLG equation is therefore modified as:

$$
\frac{d \mathbf{m}}{d t}=-\gamma \mathbf{m} \times \mathbf{H}_{\mathrm{eff}}+\alpha \mathbf{m} \times \frac{d \mathbf{m}}{d t}+\frac{\gamma}{M_{t o t}} \mathbf{N}_{\mathrm{st}},
$$

where $\mathbf{H}_{\text {eff }}$ is the total effective magnetic field acting on $\mathbf{m}$, and $\alpha=\alpha_{0}+2 \alpha^{\prime}$ is the total magnetic damping including both the bulk damping and the spin pumping enhanced damping from both interfaces.

The set of equations Eqs. (1-6) describes the charge/spin transport and magnetization dynamics in the metallic magnetic heterostructures. In many cases, the transport equations Eqs. (115) and the dynamical LLG equation Eq. (6) can be solved separately by ignoring the spin pumping contribution $\mathbf{I}_{s}^{s p}$ in $\mathbf{I}_{R}$, in which case the transport only depends on the instantaneous $\mathbf{m}$ but not on $\dot{\mathbf{m}}$. However, for thin magnetic layers the spin pumping modification cannot be neglected. It has possibly important consequences, such as a voltage induced by magnetization dynamics, anisotropic magnetic damping and susceptibility tensor, and colored thermal noise, as will become clear from the discussion below.

At first, let us calculate the static $(\dot{\mathbf{m}}=0)$ magneto conductance of a spin valve. When a bias voltage $V=\left(\mu_{L}-\mu_{R}\right) / e$ is applied, we can calculate the charge current $I=I_{L}=I_{R}$ from Eqs. (2-44), hence the magneto-conductance $G=I / V$. By setting $\mu_{N}=0$ in the spacer and assuming strong spin flips in the ferromagnets $\left(\mu_{L}^{s}=\mu_{R}^{s}=0\right.$ ), we find (with $\mathbf{m} \cdot \mathbf{m}_{0}=\cos \theta$ )

$$
G(\theta)=\frac{G_{0} g}{4}\left[1-4 p \eta(\theta) \sin ^{2} \frac{\theta}{2}\right],
$$

where $G_{0}=2 e^{2} / h$ is the conductance quantum and

$$
\eta(\theta)=\frac{p g / 4}{g \sin ^{2} \frac{\theta}{2}+2 \tilde{g}_{r} \cos ^{2} \frac{\theta}{2}+g_{s f}}
$$

is the angular dependent spin current polarization with $\tilde{g}_{r} \equiv g_{r}+2 g_{i}^{2} /\left(2 g_{r}+g_{s f}\right)$. The $G(\theta)$ above agrees with Eq. (160) in Ref. 36.

\section{CHARGE PUMPING IN SPIN VALVES}

When a voltage difference $\Delta V$ is applied over a spin valve which does not excite magnetization dynamics $(\dot{\mathbf{m}}=0)$, Eqs. (2

$$
\mathbf{N}_{s t}(\theta)=\Delta V\left[\tau_{i p}(\theta) \mathbf{m} \times \mathbf{m}_{0}+\tau_{o p}(\theta) \mathbf{m}_{0}\right] \times \mathbf{m},
$$

where $\tau_{i p}$ and $\tau_{o p}$ are the so-called (angular-dependent) torkances ${ }^{37}$ for the in-plane (Slonczewski's) component and out-of-plane (effective field) component:

$$
\tau_{i p}(\theta)=\frac{e \eta(\theta)}{2 \pi} \tilde{g}_{r} \quad \text { and } \quad \tau_{o p}(\theta)=\frac{e \eta(\theta)}{2 \pi} \frac{g_{i} g_{s f}}{2 g_{r}+g_{s f}} .
$$

When the bias polarity is chosen such that the in-plane torque in Eq. (9) works against the magnetic damping, the current flow can excite magnetization dynamics, otherwise they are suppressed.

Inversely, magnetization dynamics can induce a current flow by the spin-pumping: a moving magnetization (m) pumps a spin current (with zero charge current) into adjacent contacts, and the pumped spin current is converted into a charge current $I_{P}$ (or pumping voltage $V_{P}$ ) by a static ferromagnetic filter $\left(\mathbf{m}_{0}\right) \stackrel{17}{\underline{17}}$ In the following, we use the circuit theory described in Section $\amalg$ to derive a simple expression for the charge pumping voltage (current) induced by FMR in a spin valve. We shall study two different cases, i) when the spin valve is open, so no current flow is allowed $\left(I_{c}=0\right)$, and a pumping voltage $V_{P}$ is built up; ii) when the spin valve is closed, i.e. the two ends of the spin valve are short-circuited, so no voltage difference is allowed at the two ends $\left(\mu_{L}=\mu_{R}\right)$, and a pumping current $I_{P}$ flows.

i) open circuit - For an open circuit, the charge current vanishes: $I_{c}=0$. By solving Eqs. (1,4), we find an electric voltage $V_{P}=\left(\mu_{L}-\mu_{R}\right) / e$ due to the spin pumping current $\mathbf{I}_{s}^{s p}$ :

$$
V_{P}(\theta)=R(\theta)\left[\tau_{i p}(\theta) \mathbf{m} \times \dot{\mathbf{m}}+\tau_{o p}(\theta) \dot{\mathbf{m}}\right] \cdot \mathbf{m}_{0},
$$

with the magneto-resistance $R(\theta)=1 / G(\theta)$. Both the spin-transfer torque in Eq. (9) and the charge pumping voltage in Eq. (11) are governed by the torkances. Eq. (11) confirms the two-step process for the charge pumping: 1) spin current pumped by $\dot{\mathbf{m}}, 2$ ) charge current generated by projecting on $\mathbf{m}_{0}$. Note that Eq. (11) entails all multiple scattering in the spacer.

In Eq. (11), the charge pumping voltage is related to the torkances, which also govern the spin-transfer torque. Currently, the latter can be accessed only indirectly by its effect on the current induced magnetization dynamics

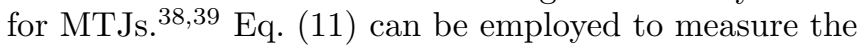
spin-transfer torque or torkances in spin valves via FMR induced voltages when the magneto-resistance $R(\theta)$ is obtained alongside as done by Urazhdin et al $\underline{\underline{40}}$

ii) closed circuit - When the spin valve is shortcircuited, $\mu_{L}=\mu_{R}$, Eqs. (114) yield a pumping current

$$
I_{P}(\theta)=\left[\tau_{i p}(\theta) \mathbf{m} \times \dot{\mathbf{m}}+\tau_{o p}(\theta) \dot{\mathbf{m}}\right] \cdot \mathbf{m}_{0} .
$$

For comparison, in the presence of an applied current current $I$,

$$
\mathbf{N}_{s t}(\theta)=R(\theta) I\left[\tau_{i p}(\theta) \mathbf{m} \times \mathbf{m}_{0}+\tau_{o p}(\theta) \mathbf{m}_{0}\right] \times \mathbf{m} .
$$

Usually, there is a passive series resistance in addition to the magneto-resistance $R(\theta)$. The charge pumping voltage for the open circuit is insensitive to such a passive resistance (for an ideal voltage meter).

In transition-metal ferromagnets, $g_{i} \leq 0.1 g_{r}, \stackrel{36}{=}$ thus from now on we neglect the imaginary part of the mixing conductance, i.e. $g_{i}=0, \tau_{o p}=0$, and $\tau_{i p}=e \eta(\theta) g_{r} / 2 \pi$. 


\section{MAGNETIZATION RELATED VOLTAGE NOISE IN SPIN VALVES}

According to the Fluctuation-Dissipation theorem (FDT), the electrical voltage fluctuations across a nonmagnetic conductor is associated with the electron linear momentum dissipation by the electrical resistance, which causes Joule heating. In ferromagnets, there are also magnetization fluctuations associated with the angular momentum dissipation or magnetic damping. In magnetic heterostructures such as spin valves, the two fluctuations (electric and magnetic) are coupled by the dynamical exchange of spin currents. Electronic noise increases the magnetic fluctuations via the spin-transfer effect. Inversely, the magnetization noise increases electronic fluctuations via spin/charge pumping.

We discussed in the previous section the pumping voltage (current) induced by an arbitrary motion of magnetization. Here the formalism is applied to the stochastic magnetization motion at thermal equilibrium: the thermal fluctuations of magnetization induce a pumping voltage (current), which on filtering by the static layer becomes a noisy voltage signal. In this section, we discuss this magnetization fluctuation-induced voltage noise $V_{M}(t)$ in a spin valve at thermal equilibrium, the power spectrum of which is the Fourier transform of its time correlation function:

$$
S_{M}(\omega)=2 \int\left\langle V_{M}(0) V_{M}(t)\right\rangle e^{-i \omega t} d t
$$

As shown by Johnson and Nyquist,23,24 the Fluctuation-Dissipation theorem (FDT) relates the noise power spectrum $S(\omega)$ to the real part of the impedance $Z(\omega)$ which characterizes the dissipation:

$$
S(\omega)=4 k_{B} T \operatorname{Re}[Z(\omega)]
$$

We may calculate the noise spectrum from both sides of the FDT, 1) by computing the time-correlation $\left\langle V_{M}(0) V_{M}(t)\right\rangle$ from the response function, then using Eq. (14); 2) by computing the frequency-dependent impedance $Z(\omega)$ of a spin valve, which consists of an electrical and a magnetic contribution: $Z(\omega)=R_{E}+$ $Z_{M}(\omega)$, then making use of the Johnson-Nyquist formula Eq. (15). The electric part $R_{E}$ gives rise to a white Johnson-Nyquist noise of $S_{E}=4 k_{B} T R_{E}$. In this section, we focus on method 1), and calculate the voltage noise spectrum for two special cases with $\mathbf{m}_{0} \| \hat{\mathbf{x}}$ (perpendicular case) and $\mathbf{m}_{0} \| \hat{\mathbf{z}}$ (parallel case). In Appendix B, we reproduce the spectrum for $\mathbf{m}_{0} \| \hat{\mathbf{x}}$ by using method 2 ).

In bulk ferromagnets, magnetic moment dissipation is parameterized by the Gilbert damping constant $\alpha_{0}$, which is associated with thermal fluctuations of the direction of the magnetization vector ${ }^{25}$ The magnetization fluctuations are caused by a fluctuating torque from the lattice, which is represented by a thermal random magnetic field $\mathbf{h}^{0}$ : $-M_{\text {tot }} \mathbf{m} \times \mathbf{h}^{0}$. The auto-correlator of $\mathbf{h}$ is 25

$$
\left\langle\gamma h_{i}^{0}(t) \gamma h_{j}^{0}(0)\right\rangle=\frac{2 \gamma \alpha_{0} k_{\mathrm{B}} T}{M_{t o t}} \delta_{i j} \delta(t)=\Sigma_{0} \delta_{i j} \delta(t)
$$

with $i, j=x, y$ (assuming that the easy axis is along $z$ ). Similarly, the ferromagnet loses energy and angular momentum by spin pumping. The magnetic damping increment $\alpha^{\prime}$ must be accompanied by a fluctuating transverse spin current (torque) $\mathbf{I}_{s}^{f l}$ from the contacts, ${ }^{29}$ which can be represented by another random magnetic field $\mathbf{h}^{\prime}$ : $\mathbf{I}_{s}^{f l}=-M_{t o t} \mathbf{m} \times \mathbf{h}^{\prime}$ with auto-correlator ${ }^{29}$

$$
\left\langle\gamma h_{i}^{\prime}(t) \gamma h_{j}^{\prime}(0)\right\rangle=\frac{2 \gamma \alpha^{\prime} k_{\mathrm{B}} T}{M_{t o t}} \delta_{i j} \delta(t)=\Sigma^{\prime} \delta_{i j} \delta(t) .
$$

$\mathbf{h}^{\prime}$ and $\mathbf{h}^{0}$ are statistically independent: $\left\langle h_{i}^{\prime} h_{j}^{0}\right\rangle=0$.

Including spin pumping from the magnetization fluctuations and the fluctuating spin current from the contacts, the total instantaneous spin current through the $\mathrm{F} \mid \mathrm{N}$ interface between the spacer and the free layer is (see Fig. 1(b)):

$$
\mathbf{I}_{s}(t)=\mathbf{I}_{s}^{s p}+\mathbf{I}_{s}^{f l}=\frac{M_{t o t}}{\gamma}\left(\alpha^{\prime} \mathbf{m} \times \dot{\mathbf{m}}-\gamma \mathbf{m} \times \mathbf{h}^{\prime}\right) .
$$

Due to the filtering by the static layer magnetization $\mathbf{m}_{0}$, the spin current $\mathbf{I}_{s}(t)$ is converted into a charge current $I_{c}(t)$ with efficiency $\eta(\theta)$. If the imaginary part of the mixing conductance is disregarded $\left(g_{i}=0\right.$ and $\left.\tau_{o p}=0\right)$, an electrical voltage $V_{M}(t)$ is given by the same expression as Eq. (11) with $\mathbf{m} \times \dot{\mathbf{m}}$ replaced by $\mathbf{m} \times \dot{\mathbf{m}}-\left(\gamma / \alpha^{\prime}\right) \mathbf{m} \times \mathbf{h}^{\prime}:$

$$
V_{M}(t)=R(\theta) \eta(\theta) \frac{2 e}{\hbar}\left[\mathbf{m}_{0} \cdot \mathbf{I}_{s}(t)\right]=W(\theta) \mathbf{m}_{0} \cdot \mathbf{f}(t)
$$

with $W(\theta)=2 e R(\theta) \eta(\theta) e M_{t o t} / \gamma \hbar$. Assuming that $\mathbf{m}$ fluctuates around the $\hat{\mathbf{z}}$-axis $(\mathbf{m} \simeq \hat{\mathbf{z}})$, f reads (to the leading order in $\mathbf{m}$ and $\mathbf{h}^{\prime}$ )

$$
\begin{aligned}
& f_{x}(t)=\gamma h_{y}^{\prime}-\alpha^{\prime} \dot{m}_{y} \\
& f_{y}(t)=-\gamma h_{x}^{\prime}+\alpha^{\prime} \dot{m}_{x} \\
& f_{z}(t)=\gamma\left(m_{y} h_{x}^{\prime}-m_{x} h_{y}^{\prime}\right)+\alpha^{\prime}\left(m_{x} \dot{m}_{y}-m_{y} \dot{m}_{x}\right) .
\end{aligned}
$$

The spectrum $S_{M}$ depends on the direction of the polarizer. We need to compute, e.g. $F_{x}(t) \equiv\left\langle f_{x}(0) f_{x}(t)\right\rangle$ when $\mathbf{m}_{0} \| \hat{\mathbf{x}}$ and $F_{z}(t) \equiv\left\langle f_{z}(0) f_{z}(t)\right\rangle$ when $\mathbf{m}_{0} \| \hat{\mathbf{z}}$. The correlators of $\mathbf{f}$ are composed of those between $\dot{\mathbf{m}}$ and/or $\mathbf{h}^{\prime}$, which in turn can be expressed by the transverse magnetic susceptibility $\chi(\omega)$ (in frequency domain) as the response to the magnetic field $\mathbf{h}=\mathbf{h}^{0}+\mathbf{h}^{\prime}+\mathbf{h}^{\prime \prime}\left(\mathbf{h}^{\prime}\right.$ and $\mathbf{h}^{\prime \prime}$ account for the random fields from the left and right interface of the free layer):

$$
\left[\begin{array}{l}
m_{x}(\omega) \\
m_{y}(\omega)
\end{array}\right]=\chi(\omega)\left[\begin{array}{l}
\gamma h_{x}(\omega) \\
\gamma h_{y}(\omega)
\end{array}\right]
$$


All correlators can be calculated from Eq. (19): $:^{41}$

$$
\begin{aligned}
& \left\langle m_{i}(t) m_{j}(0)\right\rangle=\frac{\Sigma}{\alpha} \int \frac{1}{\omega} \chi_{i j}^{-}(\omega) e^{-i \omega t} \frac{d \omega}{2 \pi}, \\
& \left\langle\dot{m}_{i}(t) m_{j}(0)\right\rangle=-\frac{\Sigma}{\alpha} \int i \chi_{i j}^{-}(\omega) e^{-i \omega t} \frac{d \omega}{2 \pi}, \\
& \left\langle\dot{m}_{i}(t) \dot{m}_{j}(0)\right\rangle=\frac{\Sigma}{\alpha} \int \omega \chi_{i j}^{-}(\omega) e^{-i \omega t} \frac{d \omega}{2 \pi},
\end{aligned}
$$

with $\Sigma=\Sigma_{0}+2 \Sigma^{\prime}$ (the factor 2 comes from the two pumping interfaces) and $\chi_{i j}^{-}(\omega)=\left[\chi_{i j}(\omega)-\chi_{j i}^{*}(\omega)\right] / 2 i$, and

$$
\begin{aligned}
& \left\langle m_{i}(t) \gamma h_{j}^{\prime}(0)\right\rangle=\Sigma^{\prime} \int \chi_{i j}(\omega) e^{-i \omega t} \frac{d \omega}{2 \pi}, \\
& \left\langle\dot{m}_{i}(t) \gamma h_{j}^{\prime}(0)\right\rangle=-\Sigma^{\prime} \int i \omega \chi_{i j}(\omega) e^{-i \omega t} \frac{d \omega}{2 \pi},
\end{aligned}
$$

By taking all correlators between $\mathbf{m}, \dot{\mathbf{m}}$, and $\mathbf{h}^{\prime}$ into account, we confirm that the DC spin/charge current vanishes at thermal equilibrium: $\left\langle\mathbf{I}_{s}(t)\right\rangle=\left\langle I_{c}(t)\right\rangle=0$ as required by the second law of thermodynamics.

When $\mathbf{m}_{0} \| \hat{\mathbf{x}}$, the voltage noise power spectrum reads

$$
S_{M}^{x}(\omega)=2 W^{2}(\pi / 2) \int_{-\infty}^{\infty} d t e^{i \omega t} F_{x}(t),
$$

Using Eqs. (20-21), we have

$$
F_{x}(t)=\Sigma^{\prime}\left[\delta(t)-\alpha^{\prime} \int \omega \chi_{y y}^{-}(\omega) e^{-i \omega t} \frac{d \omega}{2 \pi}\right] .
$$

From Eq. (23) and Eq. (22),

$$
S_{M}^{x}(\omega)=2 W^{2}(\pi / 2) \Sigma^{\prime}\left\{1-\alpha^{\prime} \omega \operatorname{Im}\left[\chi_{y y}(\omega)\right]\right\},
$$

in terms of the imaginary part of the dynamic susceptibilities, i.e. the magnetic dissipation. A measurement of the former therefore determines the latter, serving as an alternative to e.g. FMR measurements.

When $\mathbf{m}_{0} \| \hat{\mathbf{z}}, S_{M}^{z}(\omega)$ follows from Eq. (22) by the replacement $F_{x}(t) \rightarrow F_{z}(t)$ and $W(\pi / 2) \rightarrow W(0)$. According to Eq. (18c), this involves 4-point correlators, which can be reduced to 2-point correlators by Wick's theorem: $\stackrel{30,42}{\underline{4}}\langle a b c d\rangle=\langle a b\rangle\langle c d\rangle+\langle a c\rangle\langle b d\rangle+\langle a d\rangle\langle b c\rangle$. After some tedious algebra, we reach

$$
\begin{aligned}
& S_{M}^{z}(\omega)=2 W^{2}(0) \Sigma^{\prime 2}\left\{\frac{1}{\alpha^{\prime}} \sum_{i} \operatorname{Re}\left[\chi_{i i}(0)\right]-\right. \\
& \left.\int \frac{d \omega^{\prime}}{2 \pi} \frac{\omega-2 \omega^{\prime}}{\omega^{\prime}} \sum_{i, j}(-1)^{\delta_{i j}} \chi_{i j}^{-}\left(\omega^{\prime}\right) \chi_{\bar{i} \bar{j}}^{-}\left(\omega-\omega^{\prime}\right)\right\}
\end{aligned}
$$

with $\bar{x}=y$ and $\bar{y}=x$. For the anti-parallel configuration $\left(\mathbf{m}_{0} \|-\hat{\mathbf{z}}\right)$, the formula is identical to Eq. (25) except that $W(0)$ is replaced by $W(\pi)$. Note that $\chi$ for parallel and anti-parallel cases are different, as discussed in Appendix A.

With Eq. (24) and Eq. (25), the calculation of the noise power spectrum reduces to that of the magnetic susceptibility $\chi$ for the free layer magnetization. Similar to the Gilbert damping for the free layer magnetization in a spin valve, ${ }^{43} \chi$ in general depends on the magnetization configuration of the spin valve. We derive the angular dependent $\chi$ in Appendix $\mathrm{A}$.

For simplicity we continue with an isotropic form of the magnetic susceptibility for the free layer magnetization $\chi$, which includes the effect of the spin pumping enhanced damping, but not the multiple scattering of the spin pumping current within the spacer:

$$
\chi(\omega)=\frac{1}{\left(\omega_{0}-i \alpha \omega\right)^{2}-\omega^{2}}\left(\begin{array}{cc}
\omega_{0}-i \alpha \omega & -i \omega \\
i \omega & \omega_{0}-i \alpha \omega
\end{array}\right)
$$

with $\alpha=\alpha_{0}+2 \alpha^{\prime}$ and $\omega_{0}=\gamma H_{\text {eff }}$. Using this $\chi$, we find

$$
\begin{aligned}
S_{M}^{x}(\omega) & =2 W^{2}(\pi / 2) \Sigma^{\prime} \\
& \left\{1-\alpha^{\prime} \alpha \frac{\left(1+\alpha^{2}\right) \omega^{4}+\omega_{0}^{2} \omega^{2}}{\left[\left(1+\alpha^{2}\right) \omega^{2}-\omega_{0}^{2}\right]^{2}+4 \alpha^{2} \omega^{2} \omega_{0}^{2}}\right\} .
\end{aligned}
$$

A more accurate form of $S_{M}^{x}(\omega)$ (Eq. (B2) ) as calculated in Appendix $\mathrm{B}$ using circuit theory is recovered by the method here by using the angle-dependent susceptibility tensor Eq. (A11) instead of Eq. (26) in Eq. (24).

The square root of $S_{M}^{x}(\omega)$ is plotted in Fig. 2 for the parameters in Table I] where $\eta$ is replaced by its ballistic limit $p / 2$ (left ordinate). Assuming that the spin valve resistance $R$ is dominated by the interface resistances, $R \propto 1 / A$, but does not depend on the free layer thickness $d$. Considering the volume $\Omega=A d$ and $\alpha^{\prime} \propto 1 / d, \Sigma^{\prime} \propto \alpha^{\prime} / \Omega \propto 1 /\left(A d^{2}\right)$, the white noise background in $S_{M}^{x}(\omega)$ scales like $R^{2} \Omega^{2} \Sigma^{\prime} \propto 1 / A$, and thus does not depend on $d$. The dip in Fig. 2 at the FMR frequency is remarkable. Its depth is proportional to $\alpha^{\prime}$ hence inversely proportional to the free layer thickness, whereas its width is proportional to $\alpha \omega_{0}$. The constant background of the spectrum is $\sqrt{S_{M}^{x}(\omega)} \simeq 50 \mathrm{nV} / \sqrt{\mathrm{MHz}}$, whereas the dip is about $4 \mathrm{nV} / \sqrt{\mathrm{MHz}}$. For comparison, the root-mean-square of the electrical contribution to the

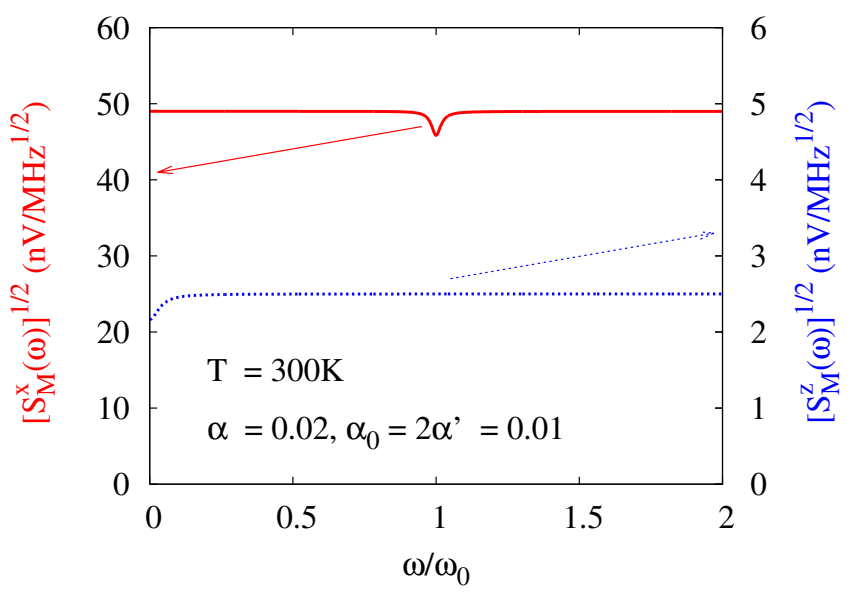

FIG. 2: (Color online) $\sqrt{S_{M}^{x}(\omega)}$ (solid line with left scale) and $\sqrt{S_{M}^{z}(\omega)}$ (dashed line with right scale). 


\begin{tabular}{r|l|r}
\hline Quantity & Values & Ref. \\
\hline \hline$M_{s}(\mathrm{Co})$ & $1.42 \times 10^{6} \mathrm{~A} \mathrm{~m}^{-1}$ & $\underline{\underline{4}}$ \\
$\gamma(\mathrm{Co})$ & $1.9 \times 10^{11}(\mathrm{~T} \mathrm{~s})^{-1}$ & $\underline{44}$ \\
$\alpha_{0}(\mathrm{Co})$ & 0.01 & $\underline{45}$ \\
$2 \alpha^{\prime}(\mathrm{Co} \mid \mathrm{Cu})$ & 0.01 & $\underline{45}$ \\
\hline$\omega_{0}$ & $10 \mathrm{GHz}$ & $\underline{46,47}$ \\
$p$ & 0.35 & $\underline{\underline{4}}$ \\
$R \simeq R_{P}$ & $0.57 \Omega$ & $\underline{\underline{4}}$ \\
$R_{\text {Sample }}$ & $1.6 \Omega$ \\
$T$ & $300 \mathrm{~K}$ & $\underline{\underline{4}}$ \\
$\Omega=A \times d$ & $\left(130 \cdot 130 \mathrm{~nm}^{2}\right) \times 2.5 \mathrm{~nm}$ & from Ref. \\
\hline${ }_{(}\left(R_{A P}-R_{P}\right) / R_{A P} \approx p^{2}$ and $R_{A P}-R_{P}=0.073 \Omega^{4}$, where P/AP \\
stands for parallel $/$ anti-parallel.
\end{tabular}

TABLE I: Typical spin valve parameters (see text).

noise is $\sqrt{S_{E}(\omega)}=\sqrt{4 k_{\mathrm{B}} T R-S_{M}^{x}(0)} \simeq 87 \mathrm{nV} / \sqrt{\mathrm{MHz}}$.

When $\mathbf{m}_{0} \| \hat{\mathbf{z}}$, Eq. (25) and Eq. (26) lead to

$$
S_{M}^{z}(\omega)=2 W^{2}(0) \frac{\Sigma^{\prime 2}}{\omega_{0}}\left[\frac{1}{\alpha^{\prime}}-\alpha \frac{\left(1+\alpha^{2}\right) \omega^{2}+4 \omega_{0}^{2}}{\left(1+\alpha^{2}\right)^{2} \omega^{2}+4 \alpha^{2} \omega_{0}^{2}}\right] .
$$

The square root of $S_{M}^{z}(\omega)$ is plotted as the dashed curve in Fig. 2 (right ordinate). In contrast to $S_{M}^{x}(\omega)$, the prefactor in $S_{M}^{z}(\omega) \propto R^{2} \Omega^{2} \Sigma^{\prime 2} / \alpha^{\prime} \propto 1 /\left(A^{2} d\right)$, therefore the noise decreases with increasing $d$. This differs from the case $\mathbf{m}_{0} \| \hat{\mathbf{x}}$ because the projection on the $\hat{\mathbf{z}}$ axis involves the average deviation of $\mathbf{m}$ from the equilibrium direction, which is inversely proportional to the volume. The divergence at vanishing thickness is caused by the neglect of the finite transparency of very thin magnetic layers for transverse spin currents. Similar to $S_{M}^{x}(\omega)$, the depth of the dip at $\omega=0$ is proportional to $\alpha^{\prime}$, hence inversely proportional to the layer thickness and has a width proportional to $\alpha \omega_{0}$.

\section{DISCUSSION}

The spectrum $S_{M}^{x}(\omega)$ consists of three contributions, which can be seen from the decomposition of $F_{x}(t)=$ $\left\langle f_{x}(t) f_{x}(0)\right\rangle=F_{x}^{s p}+F_{x}^{f l}+F_{x}^{a b}$ with

$$
\begin{aligned}
& F_{x}^{s p}(t)=\alpha^{\prime 2}\left\langle\dot{m}_{y}(t) \dot{m}_{y}(0)\right\rangle, \\
& F_{x}^{f l}(t)=\gamma^{2}\left\langle h_{y}^{\prime}(t) h_{y}^{\prime}(0)\right\rangle, \\
& F_{x}^{a b}(t)=-\alpha^{\prime} \gamma\left[\left\langle\dot{m}_{y}(t) h_{y}^{\prime}(0)\right\rangle+\left\langle\dot{m}_{y}(0) h_{y}^{\prime}(t)\right\rangle\right] .
\end{aligned}
$$

These three contributions can be interpreted as: i) a spin pumping current $F_{x}^{s p}$, which produces a peak at $\omega=\omega_{0}$, ii) a random torque (spin current) from the contact $F_{x}^{f l}$, whose spectrum is white, and iii) the absorption of the random torque from the contact by the magnetization $F_{x}^{a b}=-2 F_{x}^{s p}$, which gives a dip at $\omega=\omega_{0}$ with twice the magnitude of i). The contacts therefore provide a white-noise random torque over the ferromagnetic film, whereas the magnetization absorbs the noise power around $\omega_{0}$. The spectrum $S_{M}^{z}(\omega)$ also consists of three contributions, but the absorption line of the $\hat{\mathbf{z}}-$ component is centered at zero frequency because the fluctuations of the $\hat{\mathbf{z}}$-component magnetization do not have a characteristic frequency such as the $\hat{\mathbf{x}}, \hat{\mathbf{y}}$-components. In inhomogeneous FM films, the single macrospin mode breaks up into different eigenmodes. The noise power spectrum can then provide a "fingerprint" of the various eigenmode frequencies.

The three-point correlators arising in $\left\langle V_{M}(0) V_{M}(t)\right\rangle$ when $\mathbf{m}_{0}$ is at arbitrary angles in the $\hat{\mathbf{x}}-\hat{\mathbf{z}}$ plane vanish for normal distributions. The power spectrum is then a linear combination of $S_{M}^{x}$ and $S_{M}^{z}$, depending on the angle with dips at both the FMR and zero frequencies. The modeling of magnetic anisotropies by an easy axis is appropriate when the free layer magnetization is oriented normal to the plane in axially symmetric pillars. In standard pillars the dominant anisotropy is easy-plane, which leads to anisotropic fluctuations of the magnetization. The results remain qualitatively similar, but become anisotropic in the $\hat{\mathbf{x}}-\hat{\mathbf{y}}$ plane. For example, when a strong anisotropy constrains the fluctuations of $\mathbf{m}$ to the $\hat{\mathbf{y}}-\hat{\mathbf{z}}$ plane, $S_{M}^{y}$ vanishes. We disregarded the imaginary part of the mixing conductance in our calculation for the noise power spectrum. When it is included, the symmetric dip in the power spectrum in Fig. 2 is skewed by $g_{i}$ similar to for the spin diode effect discussed by Kupferschmidt et al. $\underline{\underline{48}}$ and Kovalev et al $\underline{32}^{32}$

For asymmetric spin valves, a non-monotonic angular dependence of the magnetoresistance and a vanishing torkance at a non-collinear magnetization configuration has been demonstrated $49,50,51,52,53$ A sign change in torkance leads to a sign change in the charge pumping voltage. The magnetic contribution to the thermal noise vanishes at the zero torkance point.

\section{SUMMARY}

In conclusion, we find that a pumping voltage arises in a spin valve when the free layer magnetization is in motion. The angular dependence of pumping voltage under FMR condition provides detailed information of the spin transport in spin valves. The pumping voltage induced by the thermal fluctuation of the free layer magnetization gives rise to additional voltage noise, which is associated with the magnetization dissipation. Thus the equilibrium electronic noise in a spin valve consists of two contributions: the Johnson-Nyquist noise associated with the fluctuations of the charge, and magnetization related noise associated with the fluctuations of the spins. The magnitude of these two contributions can be comparable. Unlike the white Johnson-Nyquist noise, the latter is found to contain an absorption line at the FMR frequency (and at zero frequency depending on the configuration) on top of an enhanced white noise back- 
ground. The noise spectrum can provide a fingerprint of the magnetic eigenmodes in inhomogeneous structures.

\section{Acknowledgment}

This work is supported by EC Contract IST-033749 "DynaMax". JX and GB thank SM for his hospitality at Tohoku University. JX acknowledges support by H. J. Gao and S. Yi in Beijing and X. F. Jin in Shanghai.

\section{APPENDIX A: ANGULAR DEPENDENCE OF THE TRANSVERSE MAGNETIC SUSCEPTIBILITY IN SPIN VALVES}

In this Appendix we calculate the transverse magnetic susceptibility for the free layer magnetization in a spin valve when no external bias is applied $\left(I_{c}=0\right)$, i.e. the only driving force is the thermal random field $\mathbf{h}$.

For an isolated magnet, the dynamics are described by the Landau-Lifshitz-Gilbert equation:

$$
\dot{\mathbf{m}}=-\gamma \mathbf{m} \times\left(\mathbf{H}_{\mathrm{eff}}+\mathbf{h}\right)+\alpha_{0} \mathbf{m} \times \dot{\mathbf{m}},
$$

with the thermal magnetic field $\mathbf{h}$ and bulk damping parameter $\alpha_{0}$. When we consider small amplitude precession around the $\hat{\mathbf{z}}$ direction (assuming $\mathbf{H}_{\text {eff }} \| \hat{\mathbf{z}}$ ), the Fourier transform of the linearized LLG equation becomes

$$
\left[\begin{array}{l}
m_{x}(\omega) \\
m_{y}(\omega)
\end{array}\right]=\chi_{0}(\omega)\left[\begin{array}{l}
\gamma h_{x}(\omega) \\
\gamma h_{y}(\omega)
\end{array}\right]
$$

with

$$
\chi_{0}(\omega)=\frac{1}{\left(\omega_{0}-i \alpha_{0} \omega\right)^{2}-\omega^{2}}\left(\begin{array}{cc}
\omega_{0}-i \alpha_{0} \omega & -i \omega \\
i \omega & \omega_{0}-i \alpha_{0} \omega
\end{array}\right) .
$$

In spin valves, $\chi$ for the free layer magnetization depends on the relative orientation of $\mathbf{m}_{0}$ and $\mathbf{m}$ because of the multiple scattering within the spacer, which depends on the orientation of $\mathbf{m}_{0}$, acts as spin-transfer torque on m. We now linearize Eq. (6), again assuming that $\mathbf{m}$ fluctuates around the $\hat{\mathbf{z}}$-axis with small amplitude $(\mathbf{m} \simeq \hat{\mathbf{z}})$ :

$$
\begin{aligned}
& \dot{m}_{x}=-\omega_{0} m_{y}-\alpha \dot{m}_{y}+\frac{\gamma}{M_{t o t}} N_{\mathrm{st}}^{x}+\gamma h_{y}, \\
& \dot{m}_{y}=+\omega_{0} m_{x}+\alpha \dot{m}_{x}+\frac{\gamma}{M_{t o t}} N_{\mathrm{st}}^{y}-\gamma h_{x},
\end{aligned}
$$

where $\alpha=\alpha_{0}+2 \alpha^{\prime}$ and $2 \alpha^{\prime}$ is the enhanced damping from the two interfaces of the free layer.

The circuit theory Eqs. (14) are coupled with the LLG equation in Eq. (A4) through $\dot{\mathbf{m}}$ in Eq. (3) and $\mathbf{I}_{R}$ in Eq. (A4) and they have to be solved self-consistently. We assume that $\mathbf{m}_{0}$ is static and tilted by an angle $\theta$ from $\hat{\mathbf{z}}$, i.e. $\hat{\mathbf{z}} \cdot \mathbf{m}_{0}=\cos \theta$. Eqs. (1, 4) can be converted to scalar equations by taking dot products with $\mathbf{m}, \mathbf{m}_{0}$, and $\mathbf{m}_{\times}=\mathbf{m} \times \mathbf{m}_{0}$. Introducing the projections of an arbitrary vector $\mathbf{q}:\left(q^{0}, q^{m}, q^{\times}\right) \equiv \mathbf{q} \cdot\left(\mathbf{m}_{0}, \mathbf{m}, \mathbf{m}_{\times}\right)$, setting $g_{s f}=0$ for simplicity, Eqs. (1-4) become

$$
\begin{aligned}
0 & =I=\frac{e g}{2 h}\left(2 \mu_{L}-p \mu_{N}^{0}\right)=-\frac{e g}{2 h}\left(2 \mu_{R}-p \mu_{N}^{m}\right) \\
I_{L}^{0} & =\frac{e g}{2 h}\left(2 p \mu_{L}-\mu_{N}^{0}\right) \\
I_{L}^{m} & =\frac{e g}{2 h}\left(2 p \mu_{L}-\mu_{N}^{0}\right) \cos \theta-\frac{e g_{r}}{h}\left(\mu_{N}^{m}-\mu_{N}^{0} \cos \theta\right) \\
I_{L}^{\times} & =-\frac{e g_{r}}{h} \mu_{N}^{\times} \\
I_{R}^{0} & =-\frac{e g}{2 h}\left(2 p \mu_{R}-g \mu_{N}^{m}\right) \cos \theta \\
& +\frac{e g_{r}}{h}\left(\mu_{N}^{0}-\mu_{N}^{m} \cos \theta\right)+\frac{e g_{r}}{2 \pi} \dot{m}^{\times} \\
I_{R}^{m} & =-\frac{e g}{2 h}\left(2 p \mu_{R}-\mu_{N}^{m}\right) \\
I_{R}^{\times} & =\frac{e}{h} g_{r} \mu_{N}^{\times}-\frac{e g_{r}}{2 \pi} \dot{m}^{0}, \\
0 & =I_{L}^{0}-I_{R}^{0}=I_{L}^{m}-I_{R}^{m}=I_{L}^{\times}-I_{R}^{\times}
\end{aligned}
$$

where in the third and fifth equation above, we disregard the time-dependence of $m^{0}(t)=\mathbf{m}(t) \cdot \mathbf{m}_{0}$ because $\mathbf{m} \simeq \hat{\mathbf{z}}$ to leading order in the deviations. The solutions to Eq. (A5) are

$$
I_{R}^{0}=\xi_{0} \frac{e g_{r}}{4 \pi} \dot{m}^{\times}, \quad I_{R}^{m}=\xi_{m} \frac{e g_{r}}{4 \pi} \dot{m}^{\times}, \quad I_{R}^{\times}=-\frac{e g_{r}}{4 \pi} \dot{m}^{0},
$$

with

$$
\xi_{0}=\frac{1-\nu}{1-\nu^{2} \cos ^{2} \theta} \quad \text { and } \quad \xi_{m}=-\frac{(1-\nu) \nu \cos \theta}{1-\nu^{2} \cos ^{2} \theta}
$$

and $\nu=\left[2 g_{r}-g\left(1-p^{2}\right)\right] /\left[2 g_{r}+g\left(1-p^{2}\right)\right]$. We now define the coordinate system in the plane normal to $\hat{\mathbf{z}}$ :

$$
\hat{\mathbf{x}} \equiv \frac{\mathbf{m}_{0}-\hat{\mathbf{z}} \cos \theta}{\sin \theta} \text { and } \hat{\mathbf{y}} \equiv \hat{\mathbf{z}} \times \hat{\mathbf{x}} \approx \frac{\mathbf{m}_{\times}}{\sin \theta} .
$$

Therefore $\dot{m}^{0} \approx \dot{m}_{x} \sin \theta, \dot{m}^{\times} \approx \dot{m}_{y} \sin \theta$, and

$$
\begin{aligned}
& \frac{\gamma}{M_{t o t}} N_{\mathrm{st}}^{x} \approx \frac{\gamma \hbar}{2 e M_{t o t}} \frac{I_{R}^{0}-I_{R}^{m} \cos \theta}{\sin \theta}=\frac{1}{2} \xi \alpha^{\prime} \dot{m}_{y}, \\
& \frac{\gamma}{M_{t o t}} N_{\mathrm{st}}^{y} \approx \frac{\gamma \hbar}{2 e M_{t o t}} \frac{I_{R}^{\times}}{\sin \theta}=-\frac{1}{2} \alpha^{\prime} \dot{m}_{x}
\end{aligned}
$$

with $\xi=\xi_{0}-\xi_{m} \cos \theta$, which is related to the angular dependent magnetic damping in Ref. 43 .

Plugging Eq. (A9) into Eq. (A4), after linearization in terms of small fluctuations about the $\hat{\mathbf{z}}$-axis and Fourier transformation, we have

$$
\left[\begin{array}{c}
\gamma h_{x}(\omega) \\
\gamma h_{y}(\omega)
\end{array}\right]=\left(\begin{array}{cc}
\omega_{0}-i \alpha_{y} \omega & i \omega \\
-i \omega & \omega_{0}-i \alpha_{x} \omega
\end{array}\right)\left[\begin{array}{l}
m_{x}(\omega) \\
m_{y}(\omega)
\end{array}\right]
$$

where $\alpha_{x}=\alpha-\frac{1}{2} \xi \alpha^{\prime}$ and $\alpha_{y}=\alpha-\frac{1}{2} \alpha^{\prime}$ reflect the damping anisotropy. From Eq. (A10, 


$$
\chi(\omega)=\left(\begin{array}{cc}
\omega_{0}-i \alpha_{y} \omega & i \omega \\
-i \omega & \omega_{0}-i \alpha_{x} \omega
\end{array}\right)^{-1}=\frac{1}{\left(1+\alpha_{x} \alpha_{y}\right) \omega^{2}-\omega_{0}^{2}+i\left(\alpha_{x}+\alpha_{y}\right) \omega_{0} \omega}\left(\begin{array}{cc}
\omega_{0}-i \alpha_{x} \omega & -i \omega \\
i \omega & \omega_{0}-i \alpha_{y} \omega
\end{array}\right) .
$$

Because $\alpha_{y}$ depends on angle $\theta$ through $\xi, \chi(\omega)$ also becomes angle dependent, i.e. the magnetic susceptibility function for the free layer magnetization in a spin valve is in general angular dependent, for the same reason as the magnetic damping in spin valves $\underline{43} \mathrm{Eq}$. (A11) reduces to Eq. (26) when we identify $\alpha_{x} \simeq \alpha_{y} \simeq \alpha$ by ignoring the back-flow correction to the damping.

\section{APPENDIX B: SPIN VALVE IMPEDANCE $Z(\omega)$ FOR $\mathbf{m}_{0} \| \hat{\mathbf{x}}$}

Here, we calculate the frequency dependence of the impedance of a spin valve by applying a small AC current at frequency $\omega: I(\omega)$. We consider the perpendicular case here, i.e. $\mathbf{m}_{0} \| \hat{\mathbf{x}}$ or $\cos \theta=0$, so that the circuit theory equations equal Eq. (A5) except that we allow for a non-vanishing charge current $I \neq 0$. Since we are now interested in the deterministic response, the thermal random fields $\mathbf{h}$ may be ignored. We can then solve Eqs. A4, A5 self-consistently in the frequency domain. We find that the impedance of the spin valve $Z(\omega)=\left[\mu_{L}(\omega)-\mu_{R}(\omega)\right] / e I(\omega)$ consists of two parts, an electric part $R_{E}$ and a magnetic part $Z_{M}(\omega): Z(\omega)=$ $R_{E}+Z_{M}(\omega)$ :

$$
\begin{aligned}
& R_{E}=\frac{4}{G_{0}} \frac{1}{g}+\eta^{2} R^{2} G_{0} g\left(1-p^{2}\right)+\eta^{2} R^{2} G_{0} g_{r}, \\
& Z_{M}^{x}=\eta^{2} R^{2} G_{0} g_{r} \times \\
& \quad\left[1-\frac{\alpha^{\prime} \omega\left(\alpha_{y} \omega+i \omega_{0}\right)}{\left(1+\alpha_{x} \alpha_{y}\right) \omega^{2}-\omega_{0}^{2}+i\left(\alpha_{x}+\alpha_{y}\right) \omega \omega_{0}}\right],
\end{aligned}
$$

where $\eta=\eta(\pi / 2)$ and $R=R(\pi / 2)$ are the polarization factor and the DC resistance for the spin valve at $\theta=\pi / 2$ or $\mathbf{m}_{0} \| \hat{\mathbf{x}} . R_{E}$ consists of the resistances associated with the electrical dissipation and half of the magnetic dissipation from the interface with the static magnetization which does not emit a spin pumping current.

By the Johnson-Nyquist formula Eq. (15), the noise spectrum $S_{M}^{x}(\omega)$ is given by

$$
S_{M}^{x}=4 k_{B} T \operatorname{Re}\left[Z_{M}^{x}(\omega)\right]=2 W^{2}(\pi / 2) \Sigma^{\prime}\left\{1-\alpha^{\prime} \frac{\alpha_{y}\left(1+\alpha_{x} \alpha_{y}\right) \omega^{4}+\alpha_{x} \omega^{2} \omega_{0}^{2}}{\left[\left(1+\alpha_{x} \alpha_{y}\right) \omega^{2}-\omega_{0}\right]^{2}+\left(\alpha_{x}+\alpha_{y}\right)^{2} \omega^{2} \omega_{0}^{2}}\right\}
$$

This equation is identical to Eq. (27) when we that the limit $\alpha_{x} \simeq \alpha_{y} \simeq \alpha$. This difference comes from the approximate form of $\chi$ in Eq. (26). If we use Eq. (A11), then the $S_{M}^{x}$ calculated from Eq. (24) will be exactly the same as Eq. (B2).
The frequency dependent impedance for $\mathbf{m}_{0} \| \hat{\mathbf{z}}$ is second order in $m_{x, y}$, therefore it is not so straightforward to calculate, which can also be seen from the non-trivial convolution in Eq. (25) calculated from magnetic susceptibility functions.
1 J. C. Slonczewski, J. Magn. Magn. Mater. 159, L1 (1996).

2 L. Berger, Phys. Rev. B 54, 9353 (1996).

3 S. I. Kiselev, J. C. Sankey, I. N. Krivorotov, N. C. Emley, R. J. Schoelkopf, R. A. Buhrman, and D. C. Ralph, Nature 425, 380 (2003).

4 J. A. Katine, F. J. Albert, R. A. Buhrman, E. B. Myers, and D. C. Ralph, Phys. Rev. Lett. 84, 3149 (2000).

5 M. Stiles and J. Miltat, Spin-Transfer Torque and Dynamics (2006), pp. 225-308.

${ }^{6}$ S. Urazhdin, N. O. Birge, W. P. Pratt, and J. Bass, Phys. Rev. Lett. 91, 146803 (2003).

7 W. H. Rippard, M. R. Pufall, S. Kaka, S. E. Russek, and T. J. Silva, Phys. Rev. Lett. 92, 027201 (2004).
8 A. Fert, V. Cros, J. M. George, J. Grollier, H. Jaffres, A. Hamzic, A. Vaures, G. Faini, J. B. Youssef, and H. L. Gall, J. Magn. Magn. Mater. 272-276, 1706 (2004).

9 J. Z. Sun, Phys. Rev. B 62, 570 (2000).

10 D. H. Hernando, Y. V. Nazarov, A. Brataas, and G. E. W. Bauer, Phys. Rev. B 62, 5700 (2000).

11 X. Waintal, E. B. Myers, P. W. Brouwer, and D. C. Ralph, Phys. Rev. B 62, 12317 (2000).

12 M. D. Stiles and A. Zangwill, Phys. Rev. B 66, 014407 (2002).

13 Z. Li and S. Zhang, Phys. Rev. B 68, 024404 (2003).

14 L. Berger, Phys. Rev. B 59, 11465 (1999).

15 X. Wang, G. E. W. Bauer, B. J. van Wees, A. Brataas, 
and Y. Tserkovnyak, Phys. Rev. Lett. 97, 216602 (2006).

16 M. V. Costache, M. Sladkov, S. M. Watts, C. H. van der Wal, and B. J. van Wees, Phys. Rev. Lett. 97, 216603 (2006).

17 J. Xiao, G. E. W. Bauer, and A. Brataas, Phys. Rev. B 77, 180407(R) (2008).

18 S. E. Barnes and S. Maekawa, Phys. Rev. Lett. 98, 246601 (2007).

19 W. M. Saslow, Phys. Rev. B 76, 184434 (2007).

${ }^{20}$ R. A. Duine, Phys. Rev. B) 77, 014409 (2008).

21 Y. Tserkovnyak and M. Mecklenburg, Phys. Rev. B 77, 134407 (2008).

22 S. A. Yang, G. S. D. Beach, C. Knutson, D. Xiao, Q. Niu, M. Tsoi, and J. L. Erskine, Phys. Rev. Lett. 102, 067201 (2009).

23 J. B. Johnson, Phys. Rev. 32, 97 (1928).

24 H. Nyquist, Phys. Rev. 32, 110 (1928).

25 W. F. Brown, Phys. Rev. 130, 1677 (1963).

26 R. H. Koch, G. Grinstein, G. A. Keefe, Y. Lu, P. L. Trouilloud, W. J. Gallagher, and S. S. P. Parkin, Phys. Rev. Lett. 84, 5419 (2000).

27 W. Wetzels, G. E. W. Bauer, and O. N. Jouravlev, Phys. Rev. Lett. 96, 127203 (2006).

28 X. Wang, W. Zhu, and D. Dimitrov, Phys. Rev. B 78, 024417 (2008).

29 J. Foros, A. Brataas, Y. Tserkovnyak, and G. E. W. Bauer, Phys. Rev. Lett. 95, 016601 (2005).

30 J. Foros, A. Brataas, G. E. W. Bauer, and Y. Tserkovnyak, Phys. Rev. B 75, 092405 (2007).

31 A. M. Deac, A. Fukushima, H. Kubota, H. Maehara, Y. Suzuki, S. Yuasa, Y. Nagamine, K. Tsunekawa, D. D. Djayaprawira, and N. Watanabe, Nature Physics 4, 803 (2008).

32 A. A. Kovalev, G. E. W. Bauer, and A. Brataas, Phys. Rev. B 75, 014430 (2007).

33 Y. Tserkovnyak, A. Brataas, and G. E. W. Bauer, Phys. Rev. Lett. 88, 117601 (2002).

34 A. Brataas, Y. V. Nazarov, and G. E. W. Bauer, Phys. Rev. Lett. 84, 2481 (2000).

35 A. Brataas, Y. Nazarov, and G. Bauer, Euro. Phys. J. B
22, 99 (2001).

36 A. Brataas, G. E. Bauer, and P. J. Kelly, Physics Reports 427, 157 (2006).

37 J. Slonczewski and J. Sun, J. Magn. Magn. Mater. 310, 169 (2007).

${ }^{38}$ H. Kubota, A. Fukushima, K. Yakushiji, T. Nagahama, S. Yuasa, K. Ando, H. Maehara, Y. Nagamine, K. Tsunekawa, D. D. Djayaprawira, et al., Nature Physics 4, 37 (2008).

39 J. C. Sankey, Y. Cui, J. Z. Sun, J. C. Slonczewski, R. A. Buhrman, and D. C. Ralph, Nature Physics 4, 67 (2008).

40 S. Urazhdin, N. O. Birge, J. Pratt, and J. Bass, Appl. Phys. Lett. 84, 1516 (2004).

41 N. Smith, J. Appl. Phys. 90, 5768 (2001).

42 K. Triantafyllopoulos, The Mathematical Scientist 28, 125 (2003).

43 Y. Tserkovnyak, A. Brataas, and G. E. W. Bauer, Phys. Rev. B 67, 140404(R) (2003).

44 S. M. Bhagat and P. Lubitz, Phys. Rev. B 10, 179 (1974).

45 J. L. Beaujour, J. H. Lee, A. D. Kent, K. Krycka, and C. Kao, Phys. Rev. B 74, 214405 (2006).

46 S. K. Upadhyay, A. Palanisami, R. N. Louie, and R. A. Buhrman, Phys. Rev. Lett. 81, 3247 (1998).

47 J. Bass and W. P. Pratt, J. Magn. Magn. Mater. 200, 274 (1999).

48 J. N. Kupferschmidt, S. Adam, and P. W. Brouwer, Phys. Rev. B 74, 134416 (2006).

49 A. A. Kovalev, A. Brataas, and G. E. W. Bauer, Phys. Rev. B 66, 224424 (2002).

50 J. Manschot, A. Brataas, and G. E. W. Bauer, Phys. Rev. B 69, 092407 (2004).

51 J. Xiao, A. Zangwill, and M. D. Stiles, Phys. Rev. B 70, 172405 (2004).

52 O. Boulle, V. Cros, J. Grollier, L. G. Pereira, C. Deranlot, F. Petroff, G. Faini, J. Barnas, and A. Fert, Nature Physics 3, 492 (2007).

53 V. S. Rychkov, S. Borlenghi, H. Jaffres, A. Fert, and X. Waintal, arXiv:0902.4360 (2009). 